\title{
Global stability behaviour for the BEK family of rotating boundary layers
}

Received: 10 June 2016 / Accepted: 5 August 2016 / Published online: 2 September 2016

(C) The Author(s) 2016. This article is published with open access at Springerlink.com

\begin{abstract}
Numerical simulations were conducted to investigate the linear global stability behaviour of the Bödewadt, Ekman, von Kármán (BEK) family of flows, for cases where a disc rotates beneath an incompressible fluid that is also rotating. This extends the work reported in recent studies that only considered the rotating-disc boundary layer with a von Kármán configuration, where the fluid that lies above the boundary layer remains stationary. When a homogeneous flow approximation is made, neglecting the radial variation of the basic state, it can be shown that linearised disturbances are susceptible to absolute instability. We shall demonstrate that, despite this prediction of absolute instability, the disturbance development exhibits globally stable behaviour in the BEK boundary layers with a genuine radial inhomogeneity. For configurations where the disc rotation rate is greater than that of the overlying fluid, disturbances propagate radially outwards and there is only a convective form of instability. This replicates the behaviour that had previously been documented when the fluid did not rotate beyond the boundary layer. However, if the fluid rotation rate is taken to exceed that of the disc, then the propagation direction reverses and disturbances grow while convecting radially inwards. Eventually, as they approach regions of smaller radii, where stability is predicted according to the homogeneous flow approximation, the growth rates reduce until decay takes over. Given sufficient time, such disturbances can begin to diminish at every radial location, even those which are positioned outwards from the radius associated with the onset of absolute instability. This leads to the confinement of the disturbance development within a finitely bounded region of the spatial-temporal plane.
\end{abstract}

Keywords Rotating flows $\cdot$ Boundary layer stability $\cdot$ Global stability $\cdot$ Vorticity-based simulation

\section{Introduction}

The study of rotating boundary layers has applications to many engineering and physical phenomena. These range across aerospace technology, meteorology and oceanography. A well-known example is the Ekman boundary layer [1], which is generated when a fluid rotates above a solid surface that is also subject to rotation. This provides an idealised basic flow state for simple models that describe the dynamics of hurricanes and cyclones [2]. Another case that has been extensively examined is the von Kármán rotating-disc boundary layer [3]. This flow arises when a disc, which is in principle unbounded, rotates beneath an otherwise stationary body of fluid. Perturbations of the flow lead to the appearance of disturbance patterns that are similar to those that

Communicated by Dr. Vassilios Theofilis.

C. Davies $(\varangle)$

School of Mathematics, Cardiff University, Cardiff CF24 4AG, UK

E-mail: DaviesC9@cardiff.ac.uk

C. Thomas

Department of Mathematics, Imperial College London, London SW7 2AZ, UK 
can be observed on the wings of aircraft [4], namely vortex structures that arise from crossflow instability. The Ekman and von Kármán flows can, by means of a continuous parameterisation using the Rossby number, be construed as belonging to a wider class of three-dimensional boundary layer systems known as the Bödewadt, Ekman, von Kármán (BEK) family of rotating flows. All of these flows are obtained for a physical configuration that involves a solid flat surface that is allowed to rotate beneath a body of fluid that may also rotate, but at a possibly different angular velocity.

The first designated member of the BEK family, the Bödewadt boundary layer flow [5], corresponds to the case where there is a rotating fluid, but the solid surface lying beneath it remains stationary. An approximate version of this flow was very aptly described by Batchelor [6], making use of the simple example of stirring sugar into a mug of tea. Deploying streamlines for illustration, Batchelor demonstrated how the introduction of rotation for the bounding surface would affect the radial motion of the fluid. When the rate at which the fluid rotates exceeds that of the surface (which, following the usual custom, we will frequently refer to as being a disc), the fluid flows in a radially inward direction. However, this radial orientation is reversed when the angular velocity of the surface is taken to be greater than that of the fluid. The Bödewadt boundary layer displays the first of these two behaviours, while the latter is associated with the von Kármán boundary layer. The Ekman layer may be thought of as marking a transition point amongst the members of the BEK family. It is generated when the rotation rates of the surface and the fluid are of similar magnitude. For this particular case, the physical configuration is such that any distinction that might be drawn between radially inward and radially outward motions becomes a matter of convention, as will be discussed when the numerical simulation results are presented.

The undisturbed base-flow velocity profiles for all members of the BEK family of flows can be represented using the same similarity structure as that which was first identified by von Kármán [3] for the rotating-disc boundary layer. In each case, there is a constant boundary layer thickness, together with radial and azimuthal velocity components which have magnitudes that increase in direct proportion to the radius, while the vertical velocity is independent of the radius. The imposition of this structure, for an incompressible flow governed by the Navier-Stokes equations, leads to a coupled set of ordinary differential equations. These can be solved, numerically, to determine the vertical variation of the flow across the boundary layer [7-9].

\section{Instability types}

Rotating boundary layers are susceptible to a variety of different kinds of instability. The first type of instability to be identified in the literature was the crossflow instability, which is promoted by an inviscid mechanism involving inflection points of the undisturbed flow profiles $[4,10,11]$. A second type of instability can be generated due to the Coriolis effects that are present in rotating flow systems [12,13]. The Coriolis mechanism is generally found to destabilise disturbances at Reynolds numbers smaller than those which correspond to the onset of the crossflow instability. However, the relatively large amplitude growth that is typically associated with the crossflow mode means that this provides the form of instability that is more often and most readily observed in physical experiments.

\subsection{Local stability theory}

When a radially homogeneous base-flow approximation is applied, in order to make the linear stability problem separable along the radial direction, the possible modes of instability may be studied by determining the solutions of a dispersion relationship that can be cast in the form

$$
D(\alpha, \omega ; \beta, R o, R e)=0,
$$

where $\alpha$ and $\beta$, respectively, denote the radial and azimuthal wavenumbers, $\omega$ is the temporal frequency, $R e$ is the Reynolds number and $R o$ is the Rossby number. The possibility of unambiguously specifying the radial wavenumber $\alpha$ only arises because of the homogeneity approximation. For perturbations in the genuine, radially varying base flow, it must be construed as being locally defined for each radial position. With this proviso, a localised linear stability analysis can proceed in the usual manner. Neutral stability curves can be constructed by determining sets of parameters for which $\alpha$ and $\omega$ are both simultaneously real. For example, the critical Reynolds numbers for stationary $(\omega=0)$ crossflow forms of disturbance have been computed as $R e \approx 27,116$ and 290 for the respective Bödewadt, Ekman and von Kármán flows [8,9,13-15]. However, as 
is well known, the determination of the evolution of disturbances from a given initial spatial distribution, or subsequent to the application of an impulsive excitation, usually requires a much more subtle investigation of the dispersion relation. This involves the tracing of singularities, exhibited as pinch points between different solution branches [16]. The flow may then be classified as being stable, convectively unstable or absolutely unstable.

The onset of absolute instability for the case of the von Kármán rotating-disc boundary layer was first identified and characterised by Lingwood [17], who found that it involved an interaction between the crossflow mode and a spatially damped mode [18]. The critical Reynolds number for absolute instability was computed as being about $507[9,17]$, which was noted to be close to the values associated with the transition to turbulence in physical experiments [19]. Absolute instability was later confirmed to persist across the whole of the BEK family of rotating boundary layers, with onset Reynolds number values given as approximately 22 and 198 for the Bödewadt and Ekman flows [9], respectively.

The physical interpretation of the local stability results depends very significantly upon the sign of the Rossby number Ro. This will be illustrated in more detail later, when we consider numerical simulations for the global disturbance development. For cases with a negative Rossby number, such as the von Kármán rotating-disc boundary layer, the convection of disturbances is predominantly in a radially outward direction, for which the local Reynolds number (which is proportional to the non-dimensional radius) is increasing. As the disturbance propagates, it encounters a flow which in general becomes, at least locally, more and more unstable. Increasing levels of growth, both temporal and spatial, may thus be anticipated once the radius associated with the critical Reynolds number for the onset of absolute instability has been exceeded. However, for positive Rossby number flows, including the Bödewadt layer, convective propagation is strongest along the radially inward direction. This means that a disturbance will tend to travel towards regions with a lower Reynolds number, where the flow is predicted to be locally more stable. Eventually, the disturbance may thus encroach upon positions for which the Reynolds number is small enough for the flow to have become subcritical for both absolute and convective forms of instability.

\subsection{Global stability behaviour}

For the von Kármán rotating-disc boundary layer, no unequivocal evidence for the presence of a linearly unstable global mode has, as yet, been adduced using data obtained from physical experiments [19,20]. Disturbances with such a form might have been expected to have arisen in conjunction with the onset of absolute instability. The absence of any modes that exhibited global temporal growth was first inferred from a theoretical investigation by Davies and Carpenter [21], who conducted numerical simulations to study the space-time evolution of linearised disturbances. They employed the exact undisturbed base flow, which was not artificially simplified by applying an approximation of radial homogeneity. It was later shown that the promotion of global stability by the radial inhomogeneity could be explained by considering the convection of disturbances between regions with varying locally defined temporal frequencies for the absolute instability [22]. This meant that there was no selection of any dominant temporal frequency. Subsequent studies considered modifications of the von Kármán rotating-disc boundary layer, to incorporate the effects of mass transfer at the disc surface [23] and axial magnetic fields [24]. The results suggested that the absence of any global behaviour characterised by a well-defined temporal frequency could be construed as being a generic feature for the linear disturbance development in all of these flows.

In the current study, we shall present numerical simulation results to address the global stability behaviour of the BEK family of rotating boundary layer flows. It will be shown that the effects arising from the radial inhomogeneity of the base flow remain globally stabilising in all of the instances considered. The only exception to this is for the limiting case of a vanishing Rossby number, namely the Ekman layer, where the base flow can be construed as being radially homogeneous without making any form of approximation. Most of the remainder of the paper will be devoted to reporting and discussing the simulation results. Before this, we first present a summary of the mathematical formulation of the governing equations, both for the base flow and for the perturbations. 


\section{Governing equations}

\subsection{Basic state}

A semi-infinite body of viscous incompressible fluid of kinematic viscosity $v^{*}$ rotates with angular velocity $\Lambda_{f}^{*}$ above a flat surface (the notional disc), which has a rotation rate $\Lambda_{d}^{*}$ about the same axis. For the Bödewadt, Ekman and von Kármán boundary layer flows, which may be construed as providing the three principal examples of such a physical configuration, the rotation rates are, respectively, defined as $\Lambda_{d}^{*}=0, \Lambda_{f}^{*} \neq 0$; $\Lambda_{d}^{*} \approx \Lambda_{f}^{*} ;$ and $\Lambda_{f}^{*}=0, \Lambda_{d}^{*} \neq 0$. An asterisk is used here to denote dimensional quantities.

Dimensionless similarity variables [3] may be chosen to write the basic states for the BEK family of flows in the general form

$$
\mathbf{U}_{\mathbf{B}}(r, z)=\left(\frac{r R o}{\operatorname{Re}} F(z), \frac{r R o}{\operatorname{Re}} G(z), \frac{\operatorname{Ro}}{\operatorname{Re}} H(z)\right)
$$

where the functions $F, G, H$ represent the respective profiles for the radial, azimuthal and wall-normal velocity components. The non-dimensional radial and wall-normal coordinates are defined as $r=r^{*} / \delta^{*}$ and $z=z^{*} / \delta^{*}$, for a constant boundary layer thickness $\delta^{*}=\left(v^{*} / \Lambda^{*}\right)^{\frac{1}{2}}$, where $\Lambda^{*}$ provides a reference rotation rate for the system [9]. The Rossby and Reynolds numbers are specified as

$$
\begin{aligned}
& R o=\frac{\Delta \Lambda^{*}}{\Lambda^{*}}, \\
& \operatorname{Re}=\frac{\left(r_{a}^{*} \Delta \Lambda^{*}\right) \delta^{*}}{\nu^{*}} \equiv r_{a} \operatorname{Ro},
\end{aligned}
$$

where $\Delta \Lambda^{*}=\Lambda_{f}^{*}-\Lambda_{d}^{*}$ is the rotation rate increment. It should be noted that the difference between the circumferential speeds for the fluid and the disc, as determined at the reference radius $r_{a}^{*}$, has been deployed to provide the velocity scale used for the non-dimensionalisation. This renders the Reynolds number to have an inherent radial localisation, except for case of the Ekman layer.

The choices $R o=1,0,-1$ correspond, in turn, to the Bödewadt, Ekman and von Kármán boundary layers. It may be observed that it is necessary to keep the ratio $R o / R e=r_{a}$ fixed and nonzero, when the Ekman layer is considered as the flow that is obtained in the limit where the Rossby number vanishes. There is also the implication that negative Rossby number flow systems must be characterised by a negative Reynolds number. However, when references are made to particular values for $R e$, only the magnitudes will be given. The radial homogeneity approximation employed in a locally based linear stability analysis amounts to simply removing the radial dependence of the base flow by artificially setting $r=r_{a}$ in the similarity solution that is given in Eq. (2). A central feature of the investigations that are to be reported here is that no such approximation is imposed, other than for code validation or for facilitating comparisons between the genuinely determined global behaviour and predictions obtained from the local stability analysis.

On substituting the base flow with the similarity structure specified by (2) into the Navier-Stokes equations, the following system of ordinary differential equations is obtained

$$
\begin{gathered}
\operatorname{Ro}\left(F^{2}+H F^{\prime}-\left(G^{2}-1\right)\right)-C o(G-1)=F^{\prime \prime}, \\
\operatorname{Ro}\left(2 F G+H G^{\prime}\right)+C o F=G^{\prime \prime}, \\
2 F+H^{\prime}=0,
\end{gathered}
$$

where a prime denotes differentiation with respect to the wall-normal direction $z$ and $C o=2 \Lambda_{d}^{*} / \Lambda^{*} \equiv$ $2-R o-R o^{2}$ is the Coriolis parameter. The system of Eq. (5) is solved subject to the boundary conditions

$$
\begin{aligned}
& F(0)=G(0)=H(0)=0, \\
& F(z \rightarrow \infty)=0, \quad G(z \rightarrow \infty)=1 .
\end{aligned}
$$




\subsection{Velocity-vorticity formulation for the disturbance evolution}

The global stability behaviour of the BEK family was investigated by conducting simulations that utilised a velocity-vorticity numerical scheme that had previously been successfully deployed for similar physical configurations [21-25]. The total velocity and vorticity fields are represented as

$$
\mathbf{U}=\mathbf{U}_{\mathbf{B}}+\mathbf{u}, \quad \boldsymbol{\Omega}=\boldsymbol{\Omega}_{\mathbf{B}}+\boldsymbol{\omega},
$$

where $\mathbf{U}_{\mathbf{B}}$ and $\boldsymbol{\Omega}_{\mathbf{B}}=\nabla \times \mathbf{U}_{\mathbf{B}}$ define the undisturbed velocity and vorticity of the basic state, while the corresponding perturbations are designated as

$$
\mathbf{u}=\left(u_{r}, u_{\theta}, u_{z}\right), \quad \boldsymbol{\omega}=\left(\omega_{r}, \omega_{\theta}, \omega_{z}\right) .
$$

Perturbation variables are then divided into two subcategories: primary $\left\{\omega_{r}, \omega_{\theta}, u_{z}\right\}$ and secondary $\left\{u_{r}, u_{\theta}, \omega_{z}\right\}$. The three primary variables are determined as solutions of the linearised governing equations

$$
\begin{aligned}
& \frac{\partial \omega_{r}}{\partial t}+\frac{1}{r} \frac{\partial N_{z}}{\partial \theta}-\frac{\partial N_{\theta}}{\partial z}-\frac{C o}{R e}\left(\omega_{\theta}+\frac{\partial u_{z}}{\partial r}\right)=\frac{1}{R e}\left(\left(\nabla^{2}-\frac{1}{r^{2}}\right) \omega_{r}-\frac{2}{r^{2}} \frac{\partial \omega_{\theta}}{\partial \theta}\right) \\
& \frac{\partial \omega_{\theta}}{\partial t}+\frac{\partial N_{r}}{\partial z}-\frac{\partial N_{z}}{\partial r}+\frac{C o}{R e}\left(\omega_{r}-\frac{1}{r} \frac{\partial u_{z}}{\partial \theta}\right)=\frac{1}{R e}\left(\left(\nabla^{2}-\frac{1}{r^{2}}\right) \omega_{\theta}+\frac{2}{r^{2}} \frac{\partial \omega_{r}}{\partial \theta}\right), \\
& \nabla^{2} u_{z}=\frac{1}{r}\left(\frac{\partial \omega_{r}}{\partial \theta}-\frac{\partial\left(r \omega_{\theta}\right)}{\partial r}\right)
\end{aligned}
$$

where

$$
\nabla^{2} f=\frac{\partial^{2} f}{\partial r^{2}}+\frac{1}{r} \frac{\partial f}{\partial r}+\frac{1}{r^{2}} \frac{\partial^{2} f}{\partial \theta^{2}}+\frac{\partial^{2} f}{\partial z^{2}},
$$

for

$$
\mathbf{N}=\left\{N_{r}, N_{\theta}, N_{z}\right\}=\boldsymbol{\Omega}_{\mathbf{B}} \times \mathbf{u}+\omega \times \mathbf{U}_{\mathbf{B}} .
$$

Equations (9a) and (9b) are the radial and azimuthal components of the vorticity transport equation in cylindrical coordinates, while Eq. (9c) is the wall-normal component of the Poisson equation that relates the velocity and the vorticity for an incompressible flow. The secondary variables are determined directly from the primary variables, by integrating across the boundary layer the equations that define the vorticity and the solenoidal condition that it must of necessity satisfy. The same process of integration also converts the no-slip conditions at the disc surface into fully equivalent integral conditions that are imposed upon the vorticity [25].

Since the current investigation only concerns the development of linear disturbances, the azimuthal invariance of the base flow allows the perturbation fields to be decomposed as

$$
\{\mathbf{u}, \boldsymbol{\omega}\}=\{\tilde{\mathbf{u}}, \tilde{\boldsymbol{\omega}}\} \mathrm{e}^{i n \theta},
$$

where $n=\bar{n} / R o=\beta R e / R o$ is the azimuthal mode number. The azimuthal wavenumber $\beta$ has commonly been treated as a continuous parameter, in the context of local stability investigations that are undertaken using a radially homogenised flow approximation. Strictly speaking, however, for global stability studies conducted without any such approximation, the mode number $n$ can only assume integer values, due to the angular periodicity of the cylindrical geometry.

\section{Numerical simulation results}

Disturbances were generated using a radially localised and impulsively applied vertical motion of the disc surface. This was implemented by deploying appropriate linearised boundary conditions, imposed at the undisturbed surface location $z=0$. (See, for example [21], for further details.) The initial forcing, taken to be narrowly centred around a given radial position $r_{e}$, was applied for a time interval that was selected to be short enough to ensure the seeding of perturbations across a wide range of temporal frequencies. These could then develop to form a disturbance wavepacket. 
Temporal frequencies and growth rates for the disturbance can be locally defined using the real and imaginary parts, respectively, of the complex-valued logarithmic derivative

$$
f=\frac{i}{A} \frac{\partial A}{\partial t} \equiv i \frac{\partial}{\partial t}(\log A)
$$

where $A$ is taken to be some measure for the amplitude. It was convenient to choose this to be the azimuthal vorticity perturbation at the disc surface $\omega_{\theta, w}$. Since the derivative defined in Eq. (13) is readily determinable at all of the radial locations and points in time that are considered in any numerical simulation, we will deploy it as a means for gauging the spatial-temporal development of the disturbance. In what follows, a multiplicative factor of the Reynolds number is incorporated and results will be presented for $f R e=\left(f_{r} R e\right)+i\left(f_{i} R e\right)$. This is in order to better facilitate comparisons between simulations conducted with and without a radial homogenisation. It amounts to introducing a global timescale, based on the system rotation rate, rather than retaining the more commonly used local timescale.

\subsection{Comparisons with localised theory for absolute instability onset}

Table 1 compares results derived from localised linear stability theory [9] with numerical simulation computations that were determined using the same artificial simplification of a radially homogenised flow. The computed temporal frequencies and growth rates were found by extracting the large time asymptotic values of logarithmic time derivatives, as defined by (13). The radial wavenumbers were determined in a similar fashion, but using spatial derivatives instead. To reduce the computational costs when tracing the parameters, simulations were only conducted for integer values of $\operatorname{Re}$ and $\bar{n}=\beta R e$. Values of $\bar{n}$ quoted from the local theory are also rounded to integers. This, inevitably, introduces some minor discrepancies.

It may be seen from the table that the computations are in very good agreement for Rossby numbers in the range of $-1 \leq R o \leq 0.6$. However, for larger values of the Rossby number, it became increasingly difficult to use the simulation data to clearly identify neutrally stable behaviour in order to demarcate the onset of absolute instability. This was due, primarily, to the highly unstable nature of the Bödewadt layer and the other similar flows with $0.6<R o \leq 1$. Convective and absolute instabilities could appear simultaneously, in such a way as to make it impracticable to unambiguously delineate between them, at least for the discretely parameterised set of time-limited simulations that it was feasible to conduct.

Table 1 Critical values for onset of absolute instability, as found by Lingwood using localised stability analysis with an homogenised flow approximation [9]

\begin{tabular}{|c|c|c|c|c|c|c|}
\hline Ro & $R e_{c}$ & $\beta_{c}$ & $\bar{n}_{c}$ & $f_{c}$ & $\alpha_{c}$ & $f_{c} R e_{c}$ \\
\hline-1.0 & 507.3 & 0.1350 & 68 & -0.0349 & $0.217-i 0.122$ & -17.71 \\
\hline-1.0 & 508 & & 68 & -0.0344 & $0.219-i 0.115$ & -17.45 \\
\hline-0.8 & 434.4 & 0.155 & 67 & -0.0391 & $-0.252-i 0.142$ & -17.0 \\
\hline-0.8 & 435 & & 67 & -0.0392 & $0.253-i 0.137$ & -17.07 \\
\hline-0.6 & 345.1 & 0.1690 & 58 & -0.0417 & $-0.294-i 0.164$ & -14.4 \\
\hline-0.6 & 346 & & 58 & -0.0416 & $0.295-i 0.153$ & -14.41 \\
\hline-0.4 & 284.5 & 0.178 & 51 & -0.0426 & $-0.329-i 0.180$ & -12.1 \\
\hline-0.4 & 285 & & 51 & -0.0418 & $0.3299-i 0.174$ & -11.92 \\
\hline-0.2 & 238.3 & 0.183 & 43 & -0.0415 & $-0.357-i 0.190$ & -9.9 \\
\hline-0.2 & 239 & & 43 & -0.0406 & $0.354-i 0.188$ & -9.70 \\
\hline 0.0 & 198.8 & 0.181 & 36 & \pm 0.0397 & $0.378 \pm \mathrm{i} 0.196$ & \pm 7.8 \\
\hline 0.0 & 198 & & 36 & \pm 0.0396 & $0.378 \pm i 0.186$ & \pm 7.85 \\
\hline 0.2 & 161.4 & 0.174 & 28 & 0.0316 & $0.393+i 0.196$ & 5.1 \\
\hline 0.2 & 162 & & 28 & 0.0308 & $0.392+i 0.191$ & 4.99 \\
\hline 0.4 & 124.6 & 0.158 & 20 & 0.0203 & $0.403+i 0.191$ & 2.5 \\
\hline 0.4 & 125 & & 20 & 0.0197 & $0.403+i 0.180$ & 2.46 \\
\hline 0.6 & 87.4 & 0.126 & 11 & -0.0022 & $0.410+i 0.176$ & -0.2 \\
\hline 0.6 & 88 & & 11 & -0.0026 & $0.403+i 0.162$ & -0.23 \\
\hline 0.8 & 51.5 & 0.051 & 3 & -0.0608 & $0.407+i 0.141$ & -3.1 \\
\hline 0.8 & - & & - & - & - & - \\
\hline 1.0 & 21.7 & -0.115 & -3 & -0.2155 & $0.341+i 0.079$ & -4.7 \\
\hline 1.0 & - & & - & - & - & - \\
\hline
\end{tabular}

Values given in italics are those determined from numerical simulations, using the same approximation. The pairs of values given for the Ekman case are associated with the distinct limits for $R o=0_{-}$and $R o=0_{+}$ 


\subsection{Negative Rossby number flows}

The archetypal configuration for negative Rossby number flows is the von Kármán rotating-disc boundary layer with $R o=-1$. As has already been mentioned, linear disturbances that develop in the genuine, radially inhomogeneous, version of this flow are believed to be globally stable [21]. Instead of growing indefinitely in time, for a given radial location, in the manner expected to be associated with absolute instability, they display a predominant convection along the radially outward direction. It might be anticipated that similar global stability characteristics would be retained for at least some of the other rotating flows comprised within the BEK family. In particular, this would be expected for flows with a negative Rossby number that is not too far removed from that for the von Kármán case. Therefore, our investigation begins by considering disturbances to the flow with Rossby number $R o=-0.8$, for which the disc rotation rate remains substantially greater than that of the fluid. (It should be recalled that only the disc is in rotation for the von Kármán boundary layer.) As is documented in Table 1, the results of a linear stability analysis for the radially homogenised flow indicate that when $R o=-0.8$, absolute instability sets in for an azimuthal mode number $\bar{n}=67$ and a critical Reynolds number that may be given, to a close approximation, as $R e_{c}=435$.

Numerical simulation results for a disturbance with $\bar{n}=67$ excited at $r_{e}=\left|R e_{c} / R o\right|=544$ are presented in Fig. 1. The plots displayed in a show how the locally determined frequencies and growth rates vary with the time $t / T$, where $T=2 \pi R e$. Solid dotted lines, labelled as $P$, are included in order to illustrate the development for the corresponding homogenised version of the flow. It may be seen that when the radial variation of the basic flow state is not taken into account, the frequency remains constant over the whole of the time interval considered. Moreover, the temporal growth rate asymptotes towards zero. This behaviour is consistent with the parameters having been chosen to be critical for the onset of absolute instability, which leads the disturbance to become dominated by neutrally stable oscillations at a single temporal frequency. The remaining plots of the computed frequencies and growth rates are for the genuine flow, which is what is of central concern here. The time variation is shown at each of four equally spaced radial positions, in the neighbourhood of the impulsive forcing location. The evolution, though very different to that found for the homogenised case, has a strong qualitative resemblance to that which was documented in previously reported studies for the von Kármán boundary layer [21]. As well as showing a marked radial dependency, the frequencies can all be seen to increase over the later parts of the given time interval, with no suggestion that they are approaching any constant value. The matching growth rates decrease, and even where they are initially positive, it may be seen that negative values are eventually attained. This indicates the setting in of temporal decay at each of the selected radial positions. It can thus be inferred that there is no unstable global mode which is triggered in response to the impulsive excitation.

The convectively unstable nature of the disturbance in the radially inhomogeneous flow is illustrated in Fig. 1b. The spatial-temporal development is displayed using amplitude contours of the azimuthal vorticity at the disc surface $\left|\omega_{\theta, w}\right|$. Leading and trailing edges of the wavepacket are readily identified. They are marked by the outer solid lines that originate from the radial centre of the applied impulse. The leading edge propagates at a large positive velocity, while the trailing edge is found to travel far more slowly, but still moves in the same radially outward direction. It would appear that the velocity associated with the trailing edge is increasing over the time period shown. By examining the plotted contours between the leading and the trailing edges, and taking account of the logarithmic spacing of the contour levels, it may be surmised that the maximum of the disturbance wavepacket is propagating radially outwards, while growing rapidly as it does so.

Figure 2a plots the temporal development of the azimuthal vorticity $\omega_{\theta, w}$ at the position of the radial centre of the impulse $r_{e}=544$ on the disc surface, for disturbances in both the homogenised and the inhomogeneous flow. Initially, similar features are displayed: there is a decrease in magnitude and the oscillations recur with a comparable frequency. However, after only a quarter of the interval that is shown, there are obvious differences between the two time histories. The magnitude of the disturbance in the homogenised flow is found to level out, while the oscillation frequency remains the same throughout the time period considered. By contrast, the disturbance in the radially inhomogeneous flow continues to decrease in amplitude and the corresponding frequency can be seen to be slowly varying in time. This concurs with what was found earlier, by considering the behaviour of the growth rates and frequencies that were defined using logarithmic derivatives. However, it provides a more immediate visualisation of the stabilising effects of the base-flow inhomogeneity.

We will now briefly present some simulation results for disturbances to the rotating flow system with $R o=-0.2$, which is a configuration where the angular velocities of the disc and fluid each have a similar magnitude. The intention is to illustrate how, over the whole range of negative Rossby number flows, the radial inhomogeneity of the base flow continues to promote global stability, with a convective form of unstable 
(a)
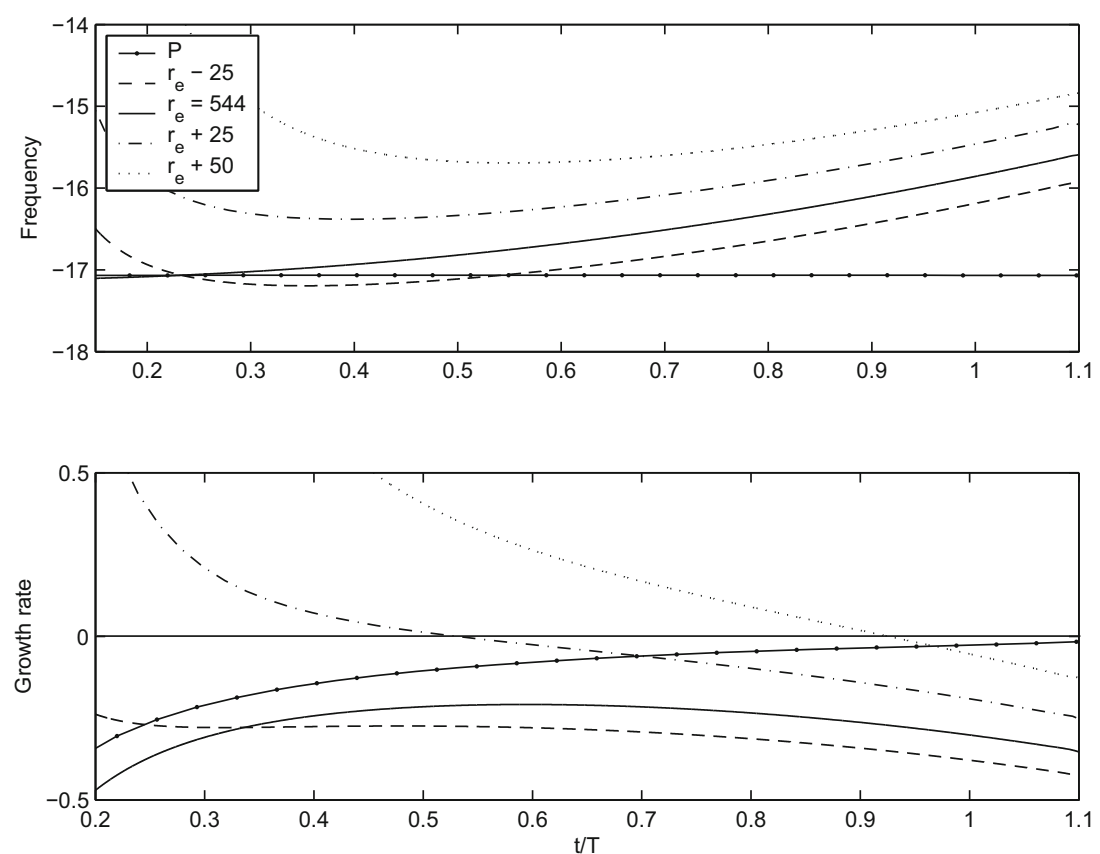

(b)

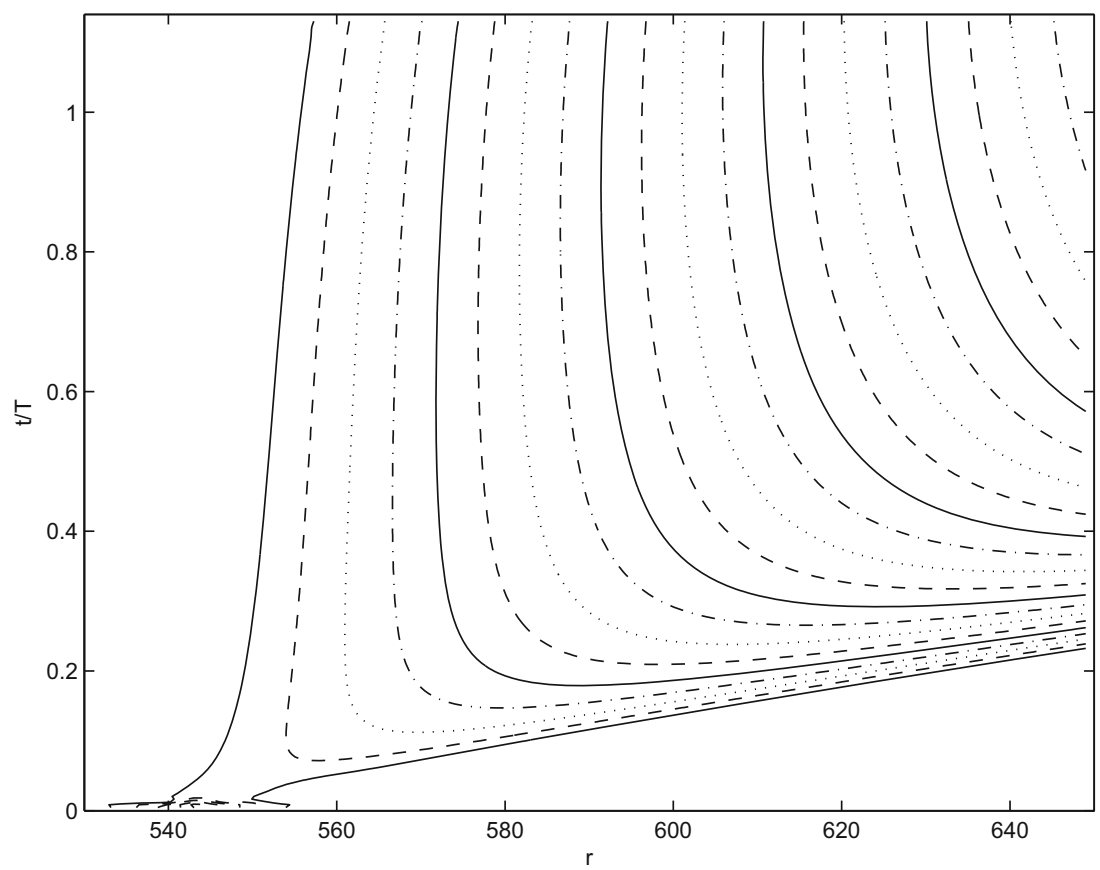

Fig. 1 a Temporal frequencies $\left(f_{r} R e\right)$ and growth rates $\left(f_{i} R e\right)$ of disturbance with $\bar{n}=67$, excited at $r_{e}=544(R e=435)$, for $R o=-0.8$. Development in genuine inhomogeneous flow at $r=r_{e}-25, r_{e}, r_{e}+25$ and $r_{e}+50$. Solid dotted lines labelled $P$ are for impulse centre in corresponding homogenised flow. b Spatial-temporal development of azimuthal vorticity at disc surface $\left|\omega_{\theta w}\right|$ for inhomogeneous flow. (Contours drawn using logarithmic scaling, as in all subsequent figures.)

behaviour remaining predominant. We again consider an excitation that is applied at the expected position for the onset of absolute instability, which in this case corresponds to a Reynolds number $R e=239$ and a radial location $r_{e}=1195$, while the azimuthal mode number is kept critical with the value $\bar{n}=43$. The temporal 

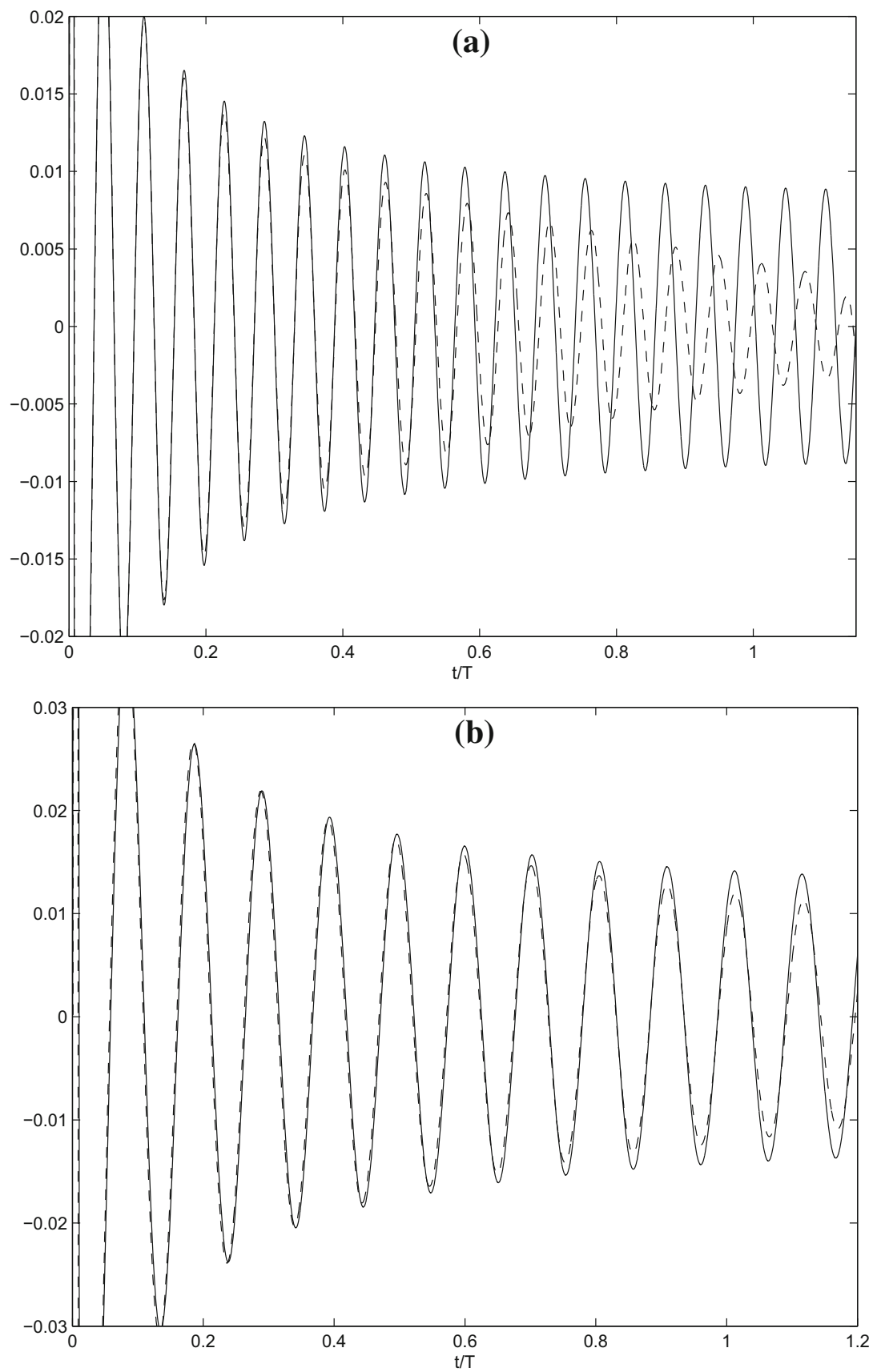

Fig. 2 Time variation of $\omega_{\theta, w}$ at centre of impulsive excitation for disturbances in homogeneous (solid line) and inhomogeneous (dashed) flows. a $R o=-0.8, \bar{n}=67, r_{e}=544(R e=435)$. b $R o=-0.2, \bar{n}=43, r_{e}=1195(R e=239)$

development of $\omega_{\theta, w}$ at $r_{e}$ is depicted in Fig. $2 \mathbf{b}$, for the artificial and exact base flows that are defined with and without the use of the radial homogeneity approximation. It can be seen that the amplitudes of the disturbances in both cases are almost identical over the entire time period shown, with small differences only appearing at the later times towards the end of the given interval. Furthermore, the oscillation frequencies displayed in the two time histories appear to remain closely comparable. Thus, the effects of the base-flow inhomogeneity would seem to have become somewhat weaker than was found for $R o=-0.8$. However, a closer examination, using the locally computed frequencies and growth rates, suggests that the same kind of qualitative behaviour has been preserved. Figure 3 displays the time variation of frequencies and growth rates for the disturbances. As before, for the genuine flow, the frequencies are radially dependent and show no discernible tendency 

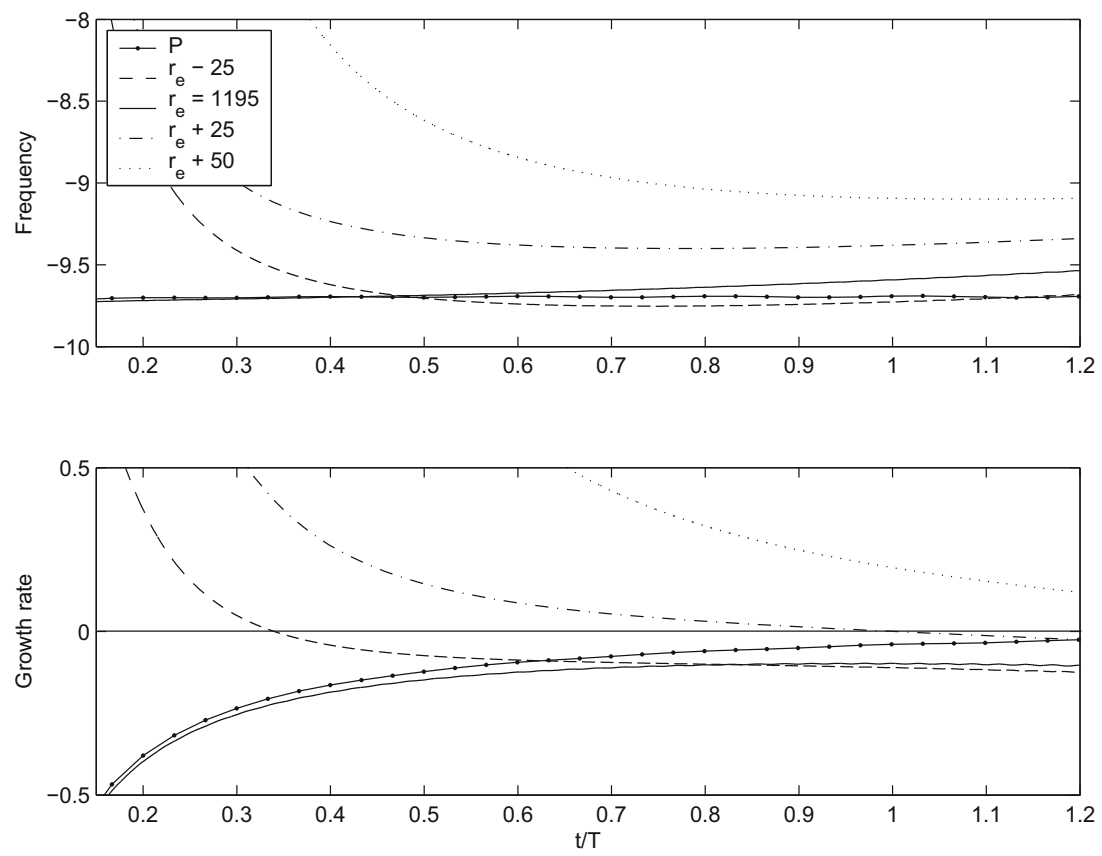

Fig. 3 Temporal frequencies and growth rates for disturbance excited at $r_{e}=1195(R e=239)$ with $\bar{n}=43$, for $R o=-0.2$. Development for inhomogeneous case at $r=r_{e}-25, r_{e}, r_{e}+25$ and $r_{e}+50$. Solid dotted lines labelled $P$ are for homogenised flow at centre of impulse

to approach a constant value. The development of the temporal growth rates indicates that, given sufficient time, decay will appear at all of the given radial locations. Though decay is not apparent at the most radially outboard position, within the time interval that is displayed, there is a clear trend towards negative values. This indicates that convectively unstable behaviour, similar to what was first found for the von Kármán flow [21], is still prevalent. It is perhaps not particularly surprising that the features that distinguish such behaviour require a longer time period to become apparent, in comparison with what occurs for rotating flows with a larger negative Rossby number. Indeed, this can be anticipated, since the Ekman layer, for which $R o=0$, is in fact strictly homogeneous, so as $R o \rightarrow 0$ the effects of inhomogeneity should diminish.

\subsection{Ekman layer}

The Ekman layer is obtained in the limit where the Rossby number vanishes. It involves no counterpart to the radial inhomogeneity that is found for all the other flows of the BEK family. This means that results obtained from the numerical simulations, and the predictions of what now becomes only a notionally localised stability theory, should exactly coincide. However, there is the interesting complication that the direction of disturbance propagation, along the coordinate that corresponds to the radius, becomes dependent upon whether the zero value of the Rossby number is considered in a limit taken from above or below. This is illustrated in Fig. 4, which depicts the spatial-temporal evolution for two essentially identical disturbances. One is for the Ekman layer with $R o=0_{-}$and the other for $R o=0_{+}$, which correspond to having the Rossby number approach zero from negative and positive values, respectively. The parameters have once more been selected to be critical for the appearance of absolute instability, which gives $R e=198$ and $\bar{n}=36$. (The impulse is centred about $r_{e}=198$, though this particular choice is made merely for convenience, since all other forcing positions would yield precisely the same behaviour, owing to the lack of any radial inhomogeneity in the base flow.) Both of the disturbances can be seen to develop in a manner that is consistent with the incipient onset of absolute instability. The leading edge of the wavepacket travels either to the left or to the right at a constant nonzero velocity, while the trailing edge becomes almost parallel to the time axis as it propagates with a diminishing and near-vanishing velocity. The disturbance convects to the right, or what can be construed as being radially outward, when $R o=0_{-}$, while it convects to the left, or radially inboard, for $R o=0_{+}$. The latter behaviour might also be expected for positive Rossby number flows, since the radial motion of the base 
(a)

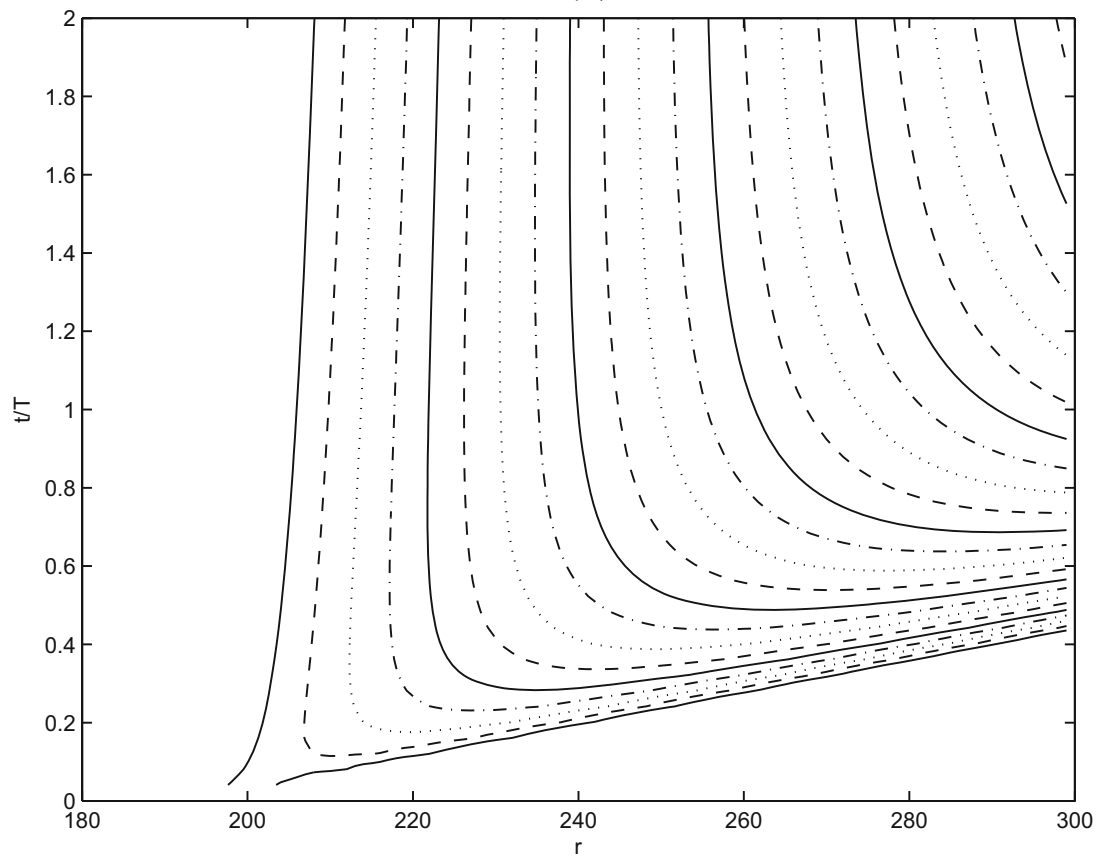

(b)

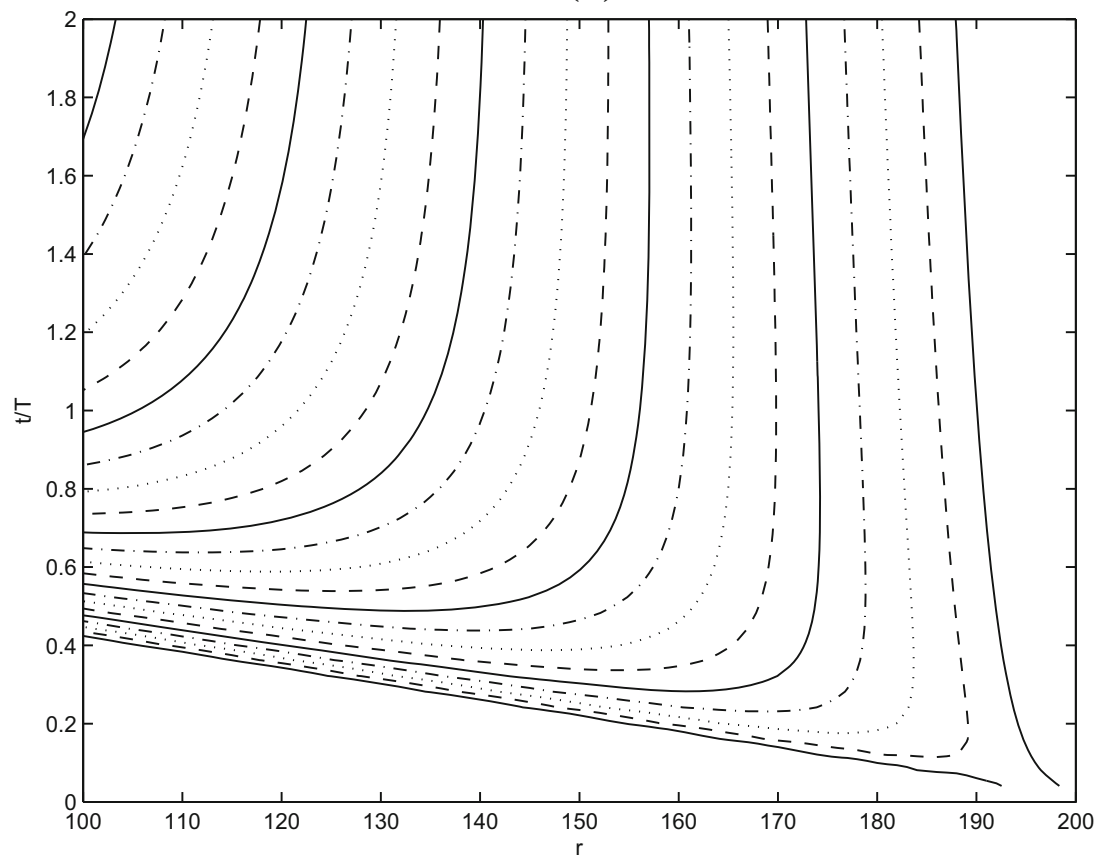

Fig. 4 Spatial-temporal development of $\left|\omega_{\theta, w}\right|$ for impulsively excited disturbances in Ekman layer, with $\bar{n}=36, R e=198$ and $r_{e}=198$. a $R o=0_{-} . \mathbf{b} R o=0_{+}$

flow reverses as $R o$ is increased through zero, and hence becomes inwardly directed towards the disc centre [6]. This expectation is entirely in accord with the reorientation of the direction for disturbance growth that is apparent in the values of the critical radial wavenumbers that are listed in Table 1. For negative Rossby numbers, these have a negative imaginary part, which is representative of spatial growth that occurs along the radially outward direction. However, for positive Rossby numbers the critical wavenumbers have a positive imaginary part, which corresponds to radially inward growth. 
(a)

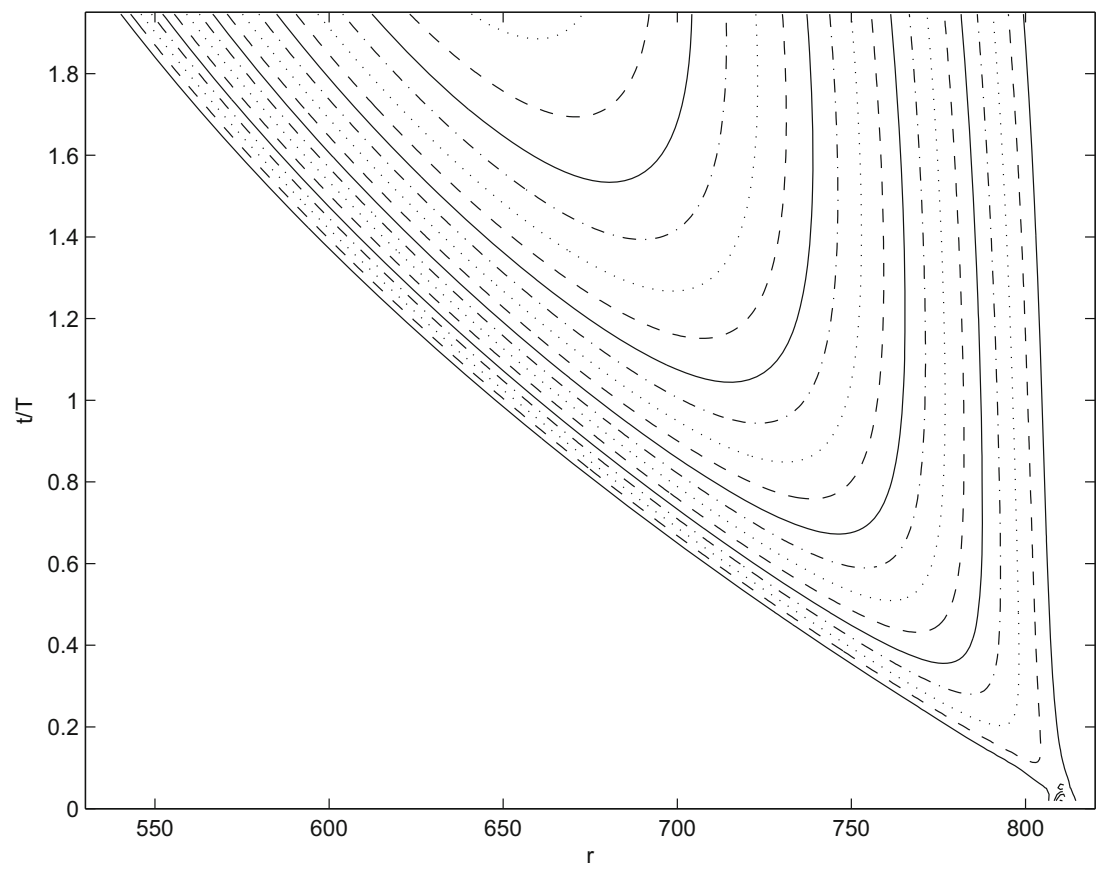

(b)

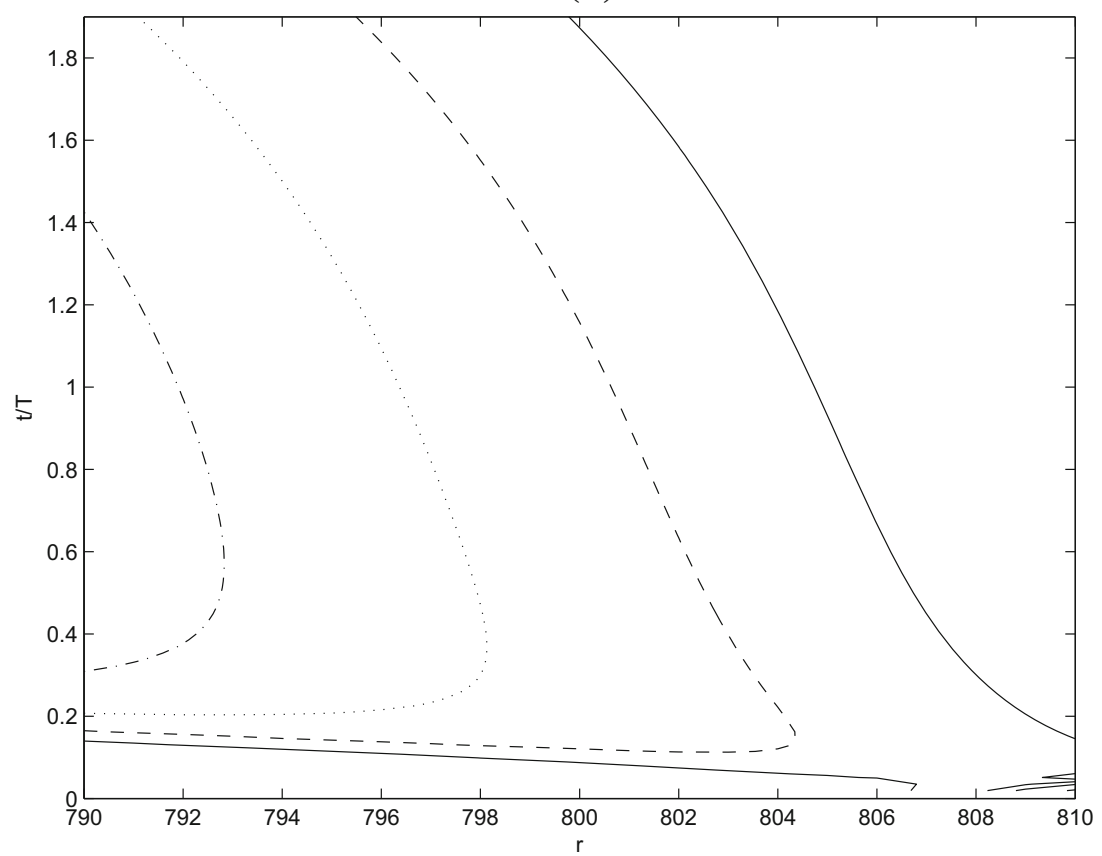

Fig. 5 Spatial-temporal development of $\left|\omega_{\theta, w}\right|$ for disturbance in inhomogeneous flow with $R o=0.2$. Impulse centred at $r_{e}=810(R e=162)$ and $\bar{n}=28$. Radial range a $530 \leq r \leq 820, \mathbf{b} 790 \leq r \leq 810$

\subsection{Positive Rossby number flows}

Figure 5a plots contours for the spatio-temporal development of a disturbance to the radially inhomogeneous rotating flow with $R o=0.2$. As for the cases considered previously, the excitation location $r_{e}=810$ ( $R e=$ 162) and the azimuthal wavenumber $\bar{n}=28$ were both chosen to coincide with the predicted onset of absolute 

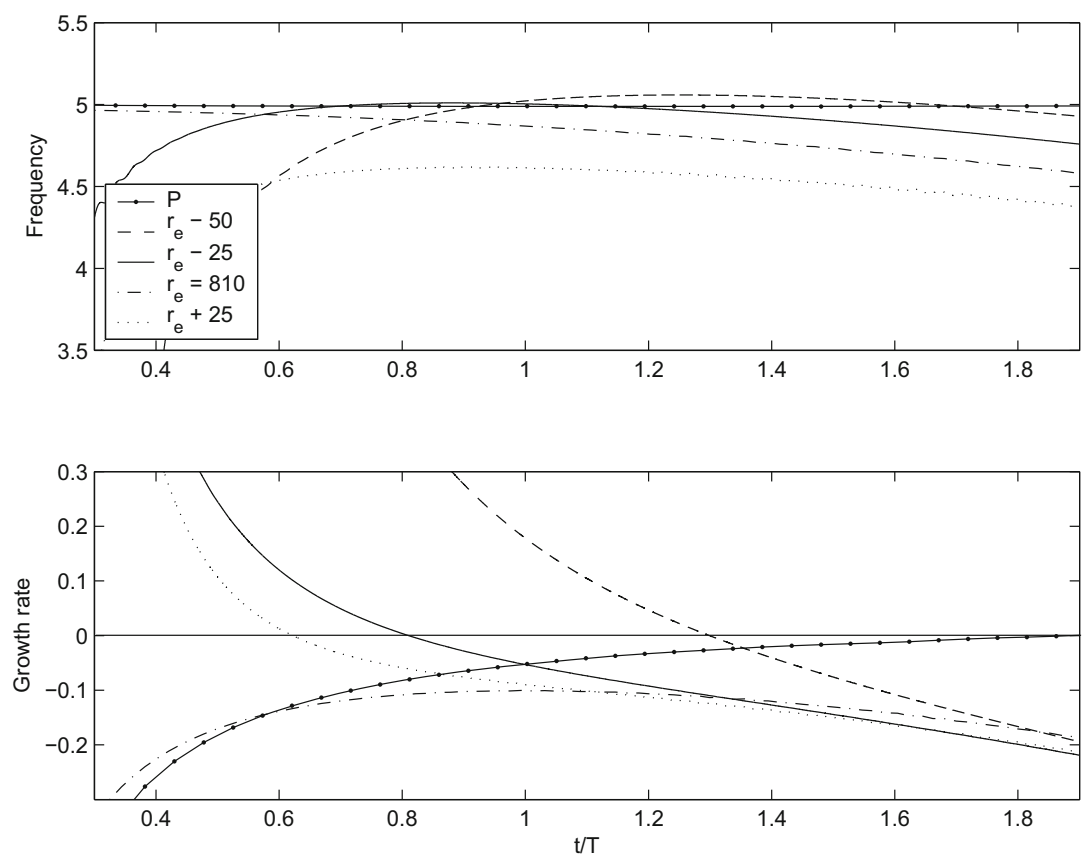

Fig. 6 Temporal frequencies and growth rates for disturbance excited at $r_{e}=810(\operatorname{Re}=162)$ with $\bar{n}=28$, for $R o=0.2$. Development for inhomogeneous case at $r=r_{e}-50, r_{e}-25, r_{e}$ and $r_{e}+25$. Solid dotted lines labelled $P$ are for homogenised flow at centre of impulse

instability. Well-defined leading and trailing edges are again clearly visible for the wavepacket. The leading edge can be immediately identified as travelling radially inwards. From an examination of the disturbance contours over the reduced radial range displayed in $\mathbf{b}$, it is apparent that the trailing edge is also propagating in an inward radial direction. Though the trailing edge moves relatively slowly, it can be seen that its velocity is increasing over time. Convection of the disturbance away from the impulse location again determines the response, although this is now directed in the radially opposite sense to that which was observed to be predominant for rotating flows with a negative Rossby number.

Computed local temporal frequencies and growth rates for the disturbance are depicted in Fig. 6. These behave in much the same fashion as has been described before. There are variations with the radial position and over time that provide little suggestion that any global mode exhibiting a well-defined temporal frequency will ever be established.

For the previously considered cases with $R o<0$, the disturbances can be construed as being unconstrained in their radially outwards convection, because of the assumption that the disc is of infinite radius. However, it may be anticipated that the inwardly directed convection of the disturbance depicted in Fig. 5 would eventually be restricted by the fact that there is, what is in effect, a fixed radial boundary at $r=0$. Moreover, the flow is predicted to become locally more and more stable as this geometrical limit is approached, since the Reynolds number reduces in proportion to the radius. In order to provide a more thorough illustration of the effects of these features, we will now focus on disturbances to the flow system with $R o=0.4$.

Increasing the value of $R o$ can be expected to strengthen the radially inward disturbance propagation. Nevertheless, because a larger Ro shifts the predicted onset of absolute instability to a lower Reynolds number and radius, it also serves to highlight the limitations on unstable convective behaviour that come into play as the disturbance propagates towards smaller radii. Initially, we will consider the case with parameters that are set to match the expected onset of absolute instability, which gives $R e=125, \bar{n}=20$ and $r_{e}=313$. For both the homogeneous and inhomogeneous flows, the spatial-temporal development of the disturbance wavepacket is plotted in Fig. 7. Behaviour that is in general accordance with critical absolute instability is clearly evident for the disturbance excited in the homogenised flow. The response that is found for the genuine radially inhomogeneous flow is very markedly different. For the earlier times following after the impulse, both edges of the disturbance wavepacket propagate radially inwards. However, at a radius of about $r=190$ and a time near to $t / T=1.8$, the leading edge reverses direction and begins to move radially outwards. Observation of the contours, for the larger disturbance amplitudes that are contained within the wavepacket, 
(a)

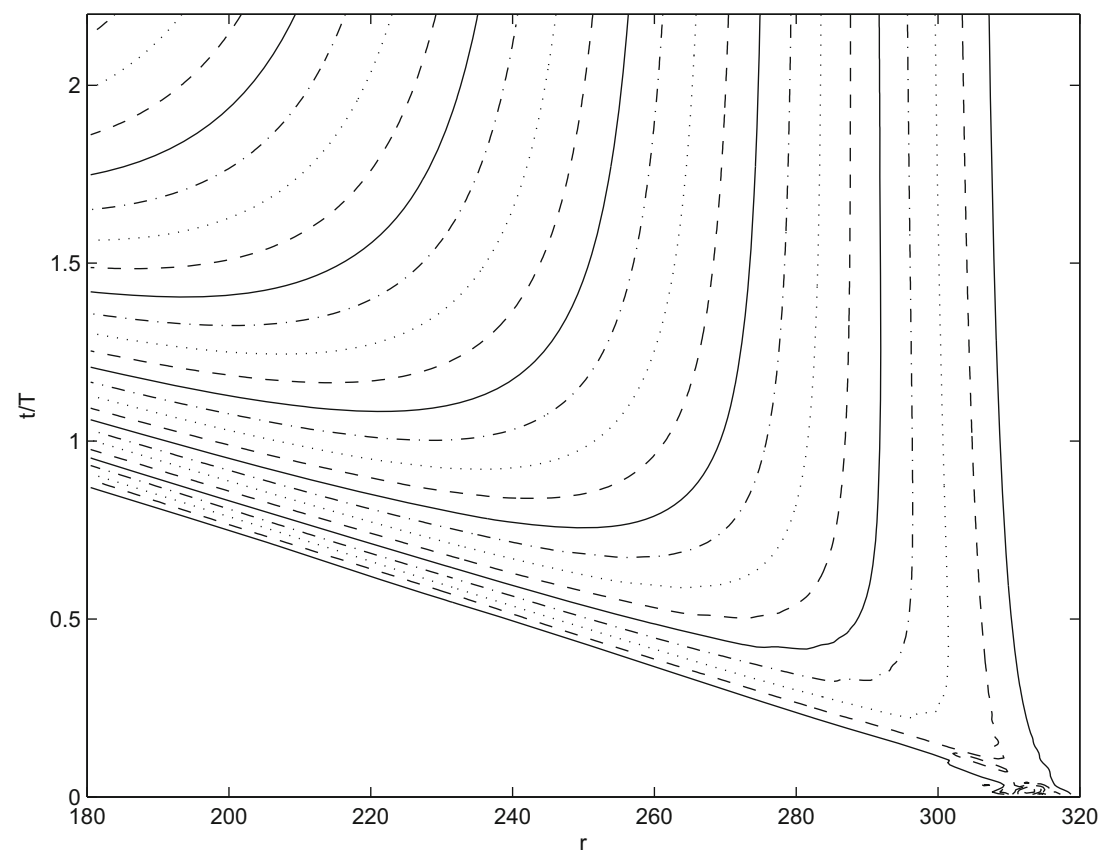

(b)

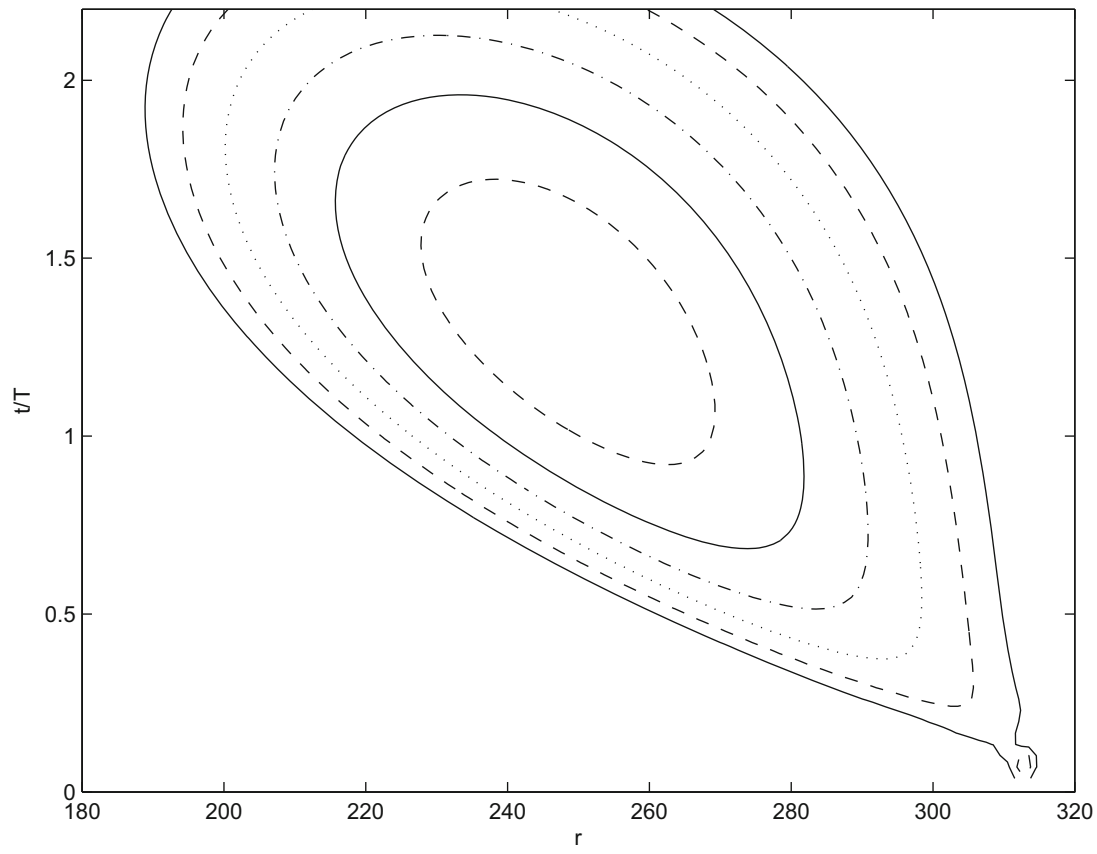

Fig. 7 a Spatial-temporal development of $\left|\omega_{\theta, w}\right|$ for disturbance in homogeneous flow for $R o=0.4$, with $R e=125$ and $\bar{n}=20$. b Corresponding wavepacket in inhomogeneous flow, with impulse centred at $r_{e}=313(\operatorname{Re}=125)$

strongly suggests that the two edges will eventually reconnect. It can be surmised that this will occur at some time around $t / T=2.5$. Thus, the region where the disturbance is displaying unstable behaviour would appear to be bounded in both space and time. For late enough times, the disturbance will have decayed at every radial location and the flow returned to a quiescent state.

In a second simulation for $R o=0.4$, the disturbance was excited at the radial position $r_{e}=375(R e=150)$, which resides well within the region of absolute instability, according to the linear stability analysis for the 
(a)

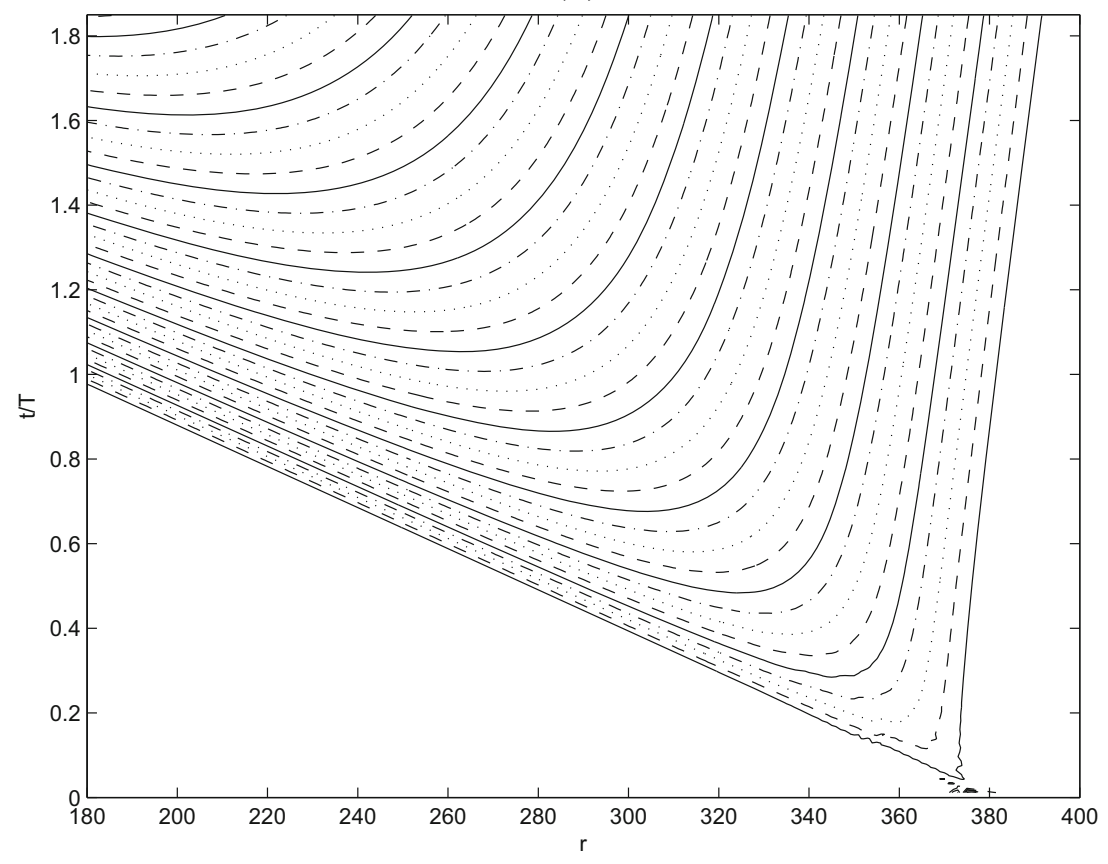

(b)

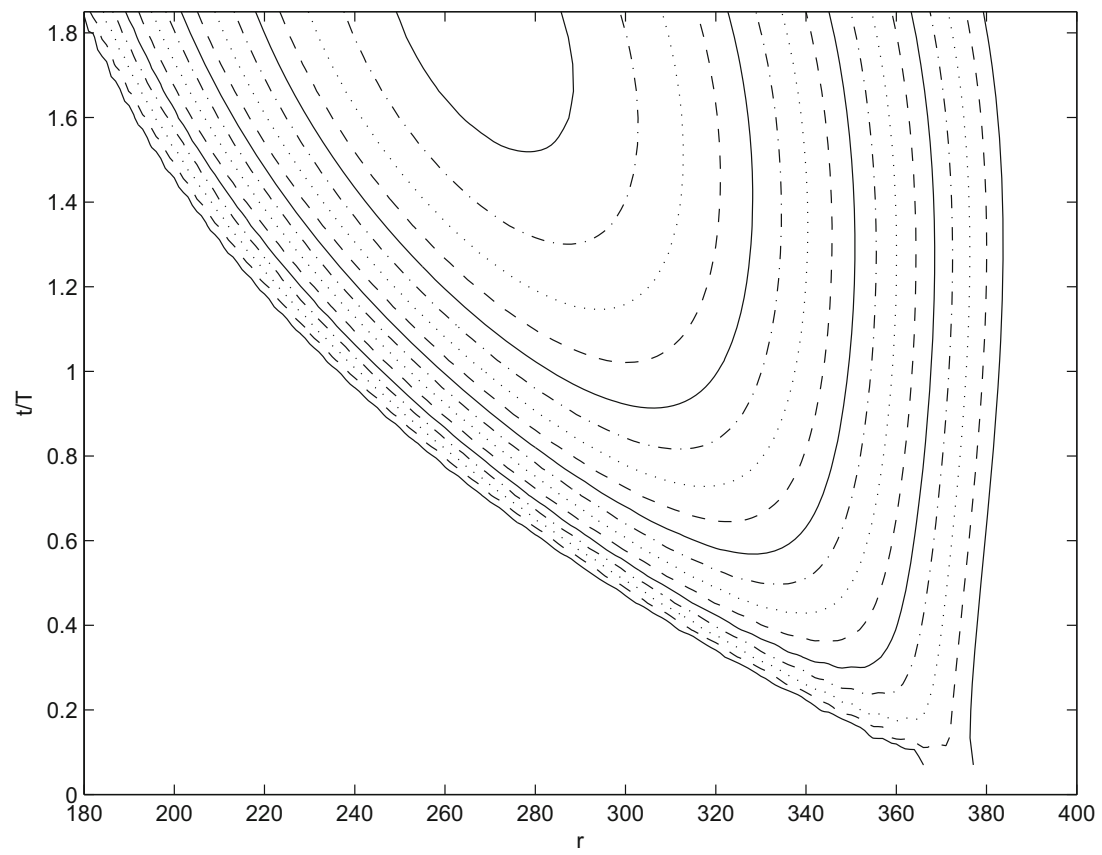

Fig. 8 a Spatial-temporal disturbance development of $\left|\omega_{\theta, w}\right|$ in homogeneous flow for $R o=0.4$, with $R e=150$ and $\bar{n}=20$. b Corresponding wavepacket in inhomogeneous flow with impulse centred at $r_{e}=375(\operatorname{Re}=150)$

homogenised flow. The spatial-temporal evolution that was determined for the inhomogeneous flow is plotted in Fig. 8, together with the corresponding homogeneous flow results, which are included for comparison. For the genuine flow, the leading and trailing edges of the wavepacket initially propagate in opposite directions. As can be seen from the plot for the homogenised case, this is what might be expected, given that the disturbance originates at a radial location which is supercritical for the emergence of absolutely unstable behaviour. Nevertheless, as times passes, it becomes apparent that strong effects are arising from the radial inhomogeneity. 

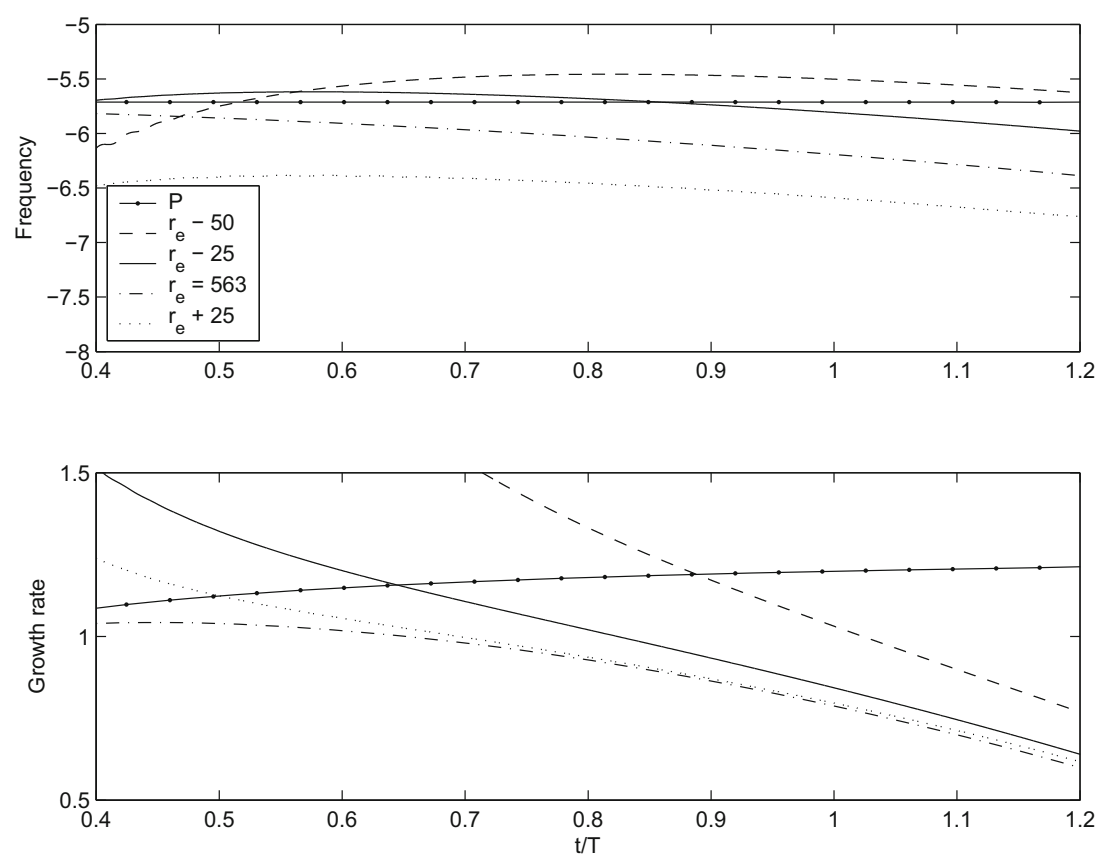

Fig. 9 Temporal frequencies and growth rates for disturbance in $R o=0.4$ flow, excited at $r_{e}=563(R e=225)$ with $\bar{n}=20$. Development in inhomogeneous flow at $r=r_{e}-50, r_{e}-25, r_{e}$ and $r_{e}+25$. Solid lines labelled $P$ are for corresponding homogeneous flow at impulse centre

At later times, the trailing edge reverses direction and begins to move radially inwards. Moreover, the velocity associated with the leading edge decreases. An examination of the contours for the higher disturbance amplitudes suggests that this edge will also eventually change direction and start to propagate radially outward. Thus, the two edges of the wavepacket would again reconnect, forming a bounded region of space and time that fully encloses the disturbance.

Figure 9 displays plots for the temporal frequencies and growth rates, taken from a third simulation of a disturbance evolving in the rotating flow with $R o=0.4$. The impulse is now applied about the radial position $r_{e}=563$, which is located very far outboard from where the onset of absolute instability is predicted. The growth rate found for the corresponding disturbance in the homogeneous flow is relatively large, so rapidly growing perturbations are to be expected. However, the temporal growth rates associated with the inhomogeneous flow are once again found to decrease. Although all of the plotted growth rates remain positive over the entire time period considered, their behaviour provides a clear indication that, given sufficient time, temporal decay will eventually set in. Hence, it would appear likely that the disturbance to the genuine flow will remain spatially temporally bounded, in a similar manner to what was seen before. This may still be the case, even though the excitation is applied at a radial position where the temporal growth for the equivalent perturbation in the homogeneous flow is so pronounced that it is conceivable that a radically different form of behaviour could appear. Unfortunately, it has not proved possible to make an entirely certain statement about such matters. In order to conduct the simulation for a sufficiently long time, and hence make a more definite judgement about the eventual disturbance behaviour, it would be necessary to overcome some numerical difficulties with the simulation method. These could usually be traced to a contamination by grid-scaled noise that was prone to becoming artificially amplified at the radial boundaries of the computational domain.

For the same reason of avoiding the introduction of any numerical artefacts, we have chosen to limit the times for which the results from our other simulations conducted for $R o=0.4$ have been reported. For larger values of the Rossby number, spurious numerical effects became much more difficult to eliminate. Moreover, as has already been mentioned earlier, when the results presented in Table 1 were first discussed, the interpretation of the spatial and temporal behaviour displayed in the simulation results became less clear for $R o>0.6$, even for the homogenised flows. Thus, we have refrained from presenting results for any such cases, preferring instead to limit our documentation to configurations where we could be confident not only of the validity of the simulation results, but also of our classification of the behaviour that was exemplified in them. 


\section{Conclusions and modelling}

The BEK family of rotating boundary layer flows has been investigated for the response to impulsively applied excitation. Globally stable behaviour, similar to that which had previously been observed for the von Kármán rotating-disc boundary layer, was found for all of the BEK flows that it proved feasible to study. However, we have only considered results for flows in the range of $-1 \leq R o \leq 0.6$. There remains the possibility that a different form of behaviour may set in as the upper limit at $R o=1$ is approached, which corresponds to the Bödewadt boundary layer.

For flows with a larger disc angular velocity than that of the overlying fluid, namely those which are parameterised by a negative Rossby number, the disturbances displayed a marked tendency to propagate radially outwards. This convection dominated the long-term response. There was no selection of a welldefined temporal frequency, as would be the case for the absolutely unstable behaviour that is predicted for the artificially homogenised version of the flow.

Similar characteristics were retained for the flows with positive Rossby numbers, where the imposed fluid rotation is taken to be greater than that of the disc. For the instances that were fully investigated, convective behaviour was again found to dominate the global response. However, the disturbance propagation was now oriented radially inwards, in the opposite sense to that which was found for negative Rossby numbers. This meant that, over time, the disturbances were convected into regions that, due to the reduction in the locally defined Reynolds number, became increasingly stable. Eventually, it appeared that temporal decay was prevalent at all radial locations, which would lead the flow to return to its original undisturbed state.

For the von Kármán rotating-disc boundary layer, as well as for some closely related variants that are subject to passive forms of flow control, it has been found that the disturbance behaviour can be successfully modelled using relatively simple impulse solutions of the linearised Ginzburg-Landau equation [22-24,26]. It is possible to deploy these to explain how global stability can be promoted, by considering the so-called detuning between the temporal frequencies predicted for the absolutely unstable responses at different radial positions. Using the same type of modelling for the other members of the BEK family that were considered in the present study, we have concluded that the globally stable nature of the disturbance behaviour can still be explained by the effects of detuning.

When considering positive Rossby number flows, it was straightforward to adapt the impulse solutions of the Ginzburg-Landau equation in order to reproduce the novel spatial-temporal confinement features that were described earlier. These emerged along with the predominance of radially inward disturbance convection. The only significant amendment in the model was an alteration in the sign of a single stability parameter, deployed in accounting for the radial growth or decay of the disturbances. This parameter is given by the imaginary part of the critical radial wavenumber for the onset of absolute instability, which as we have previously noted, becomes positive for $R o>0$. With the incorporation of such a deceptively simple change, the spatial-temporal bounds that were discovered for the disturbance development could be matched by the model solutions, yielding not only qualitative, but also a good degree of quantitative, agreement with the simulation results.

Open Access This article is distributed under the terms of the Creative Commons Attribution 4.0 International License (http:// creativecommons.org/licenses/by/4.0/), which permits unrestricted use, distribution, and reproduction in any medium, provided you give appropriate credit to the original author(s) and the source, provide a link to the Creative Commons license, and indicate if changes were made.

\section{References}

1. Ekman, V.W.: On the influence of the earth's rotation on ocean currents. Ark. Mat. Astr. Fys. 2, 1-53 (1905)

2. Nolan, D.S.: Instabilities in hurricane-like boundary-layers. Dyn. Atmos. Oceans 40, 209-236 (2005)

3. von Kármán, T.: Über laminare und turbulente Reibung. Z. Angew. Math. Mech. 1, 233-252 (1921)

4. Gregory, N., Stuart, J.T., Walker, W.S.: On the stability of three-dimensional boundary layers with application to the flow due to a rotating disk. Philos. Trans. R. Soc. Lond. Ser. A. 248, 155-199 (1955)

5. Bödewadt, U.T.: Die drehstromung uber festem grund. Z. Angew. Math. Mech. 20, 241-253 (1940)

6. Batchelor, G.K.: Note on a class of solutions of the Navier-Stokes equations representing steady rotationally-symmetric flow. Q. J. Mech. Appl. Math. 4, 29-41 (1951)

7. Rogers, M.H., Lance, G.N.: The rotationally symmetric flow of a viscous fluid in the presence of an infinite rotating disk. J. Fluid Mech. 7, 617-631 (1960)

8. Faller, A.: Instability and transition of disturbed flow over a rotating disk. J. Fluid Mech. 230, 245-269 (1991) 
9. Lingwood, R.J.: Absolute instability of the Ekman layer and related rotating flows. J. Fluid Mech. 331, 405-428 (1997)

10. Gray, W.E.: The nature of the boundary layer at the nose of a swept back wing. Unpublished, Min. Aviation, London (1952)

11. Faller, A.: An experimental study of the instability of the laminar Ekman boundary layer. J. Fluid Mech. 15, 560-576 (1963)

12. Faller, A.J., Kaylor, R.E.: A numerical study of the instability of the laminar Ekman boundary-layer. J. Atmos. Sci. 23, 466-480 (1966)

13. Malik, M.R.: The neutral curve for stationary disturbances in rotating-disk flow. J. Fluid Mech. 164, $275-287$ (1986)

14. Lilly, D.K.: On the instability of Ekman boundary flow. J. Atmos. Sci. 23, 481-494 (1966)

15. Melander, M.V.: An algorithmic approach to the linear stability of the Ekman layer. J. Fluid Mech. 132, $283-293$ (1983)

16. Briggs, R.J.: Electron-Stream Interactions in Plasmas. MIT Press, Cambridge (1964)

17. Lingwood, R.J.: Absolute instability of the boundary layer on a rotating disk. J. Fluid Mech. 183, 77-94 (1995)

18. Mack, L. M.: The Wave Pattern Produced by Point Source on a Rotating Disk. AIAA Paper 85-0490 (1985)

19. Lingwood, R.J.: An experimental study of absolute instability of the rotating-disk boundary-layer flow. J. Fluid Mech. 314, 373-405 (1996)

20. Othman, H., Corke, T.C.: Experimental investigation of absolute instability of a rotating-disk boundary layer. J. Fluid Mech. 565, 63-94 (2006)

21. Davies, C., Carpenter, P.W.: Global behaviour corresponding to the absolute instability of the rotating-disk boundary layer. J. Fluid Mech. 486, 287-329 (2003)

22. Davies, C., Thomas, C., Carpenter, P.W.: Global stability of the rotating disc boundary layer. J. Eng. Math. 57, 219-236 (2007)

23. Thomas, C., Davies, C.: The effects of mass transfer on the global stability of the rotating-disk boundary layer. J. Fluid Mech. 663, 401-433 (2010)

24. Thomas, C., Davies, C.: Global stability of the rotating-disc boundary layer with an axial magnetic field. J. Fluid Mech. 724, 510-526 (2013)

25. Davies, C., Carpenter, P.W.: A novel velocity-vorticity formulation of the Navier-Stokes equations with applications to boundary layer disturbance evolution. J. Comput. Phys. 172, 119-165 (2001)

26. Hunt, R.E., Crighton, D.G.: Instability of flows in spatially developing media. Proc. R. Soc. Lond. Ser. A. 435, 109-128 (1991) 